\title{
Utilizing network pharmacology to explore the underlying mechanism of Cinnamomi Ramulus in Zika Fever induced by Zika Virus
}

\section{Qiong Zhang}

Guangzhou University of Chinese Medicine

\section{Xuemei Lin}

Guangzhou University of Chinese Medicine

Yanni Lai

Guangzhou University of Chinese Medicine

Jiangtao Xu

Guangzhou University of Chinese Medicine

Yuting Chen

Guangzhou University of Chinese Medicine

Xiao Wang

Guangzhou University of Chinese Medicine

Xiaohong Liu

Guangzhou University of Chinese Medicine

Li geng ( $\sim$ lg@gzucm.edu.cn )

\section{Research}

Keywords: Zika fever, Cinnamomi ramulus, Network pharmacology, Inflammation

Posted Date: June 4th, 2020

DOl: https://doi.org/10.21203/rs.3.rs-32117/v1

License: (c) (i) This work is licensed under a Creative Commons Attribution 4.0 International License.

Read Full License 


\title{
Utilizing network pharmacology to explore the underlying mechanism of Cinnamomi Ramulus in Zika Fever induced by Zika Virus
}

Qiong Zhang ${ }^{1+}$, Xuemei Lin ${ }^{1+}$, Lai Yanni ${ }^{2+}$, Jiangtao $\mathrm{Xu}^{1}$, Yuting Chen ${ }^{2}$, Xiao Wang ${ }^{1 *}$, Xiaohong Liu ${ }^{1 *}$, Geng Li ${ }^{1 *}$

${ }^{1}$ Laboratory Animal Center, Guangzhou University of Chinese Medicine, Guangzhou, 510405, China

${ }^{2}$ The First Clinical Medical College, Guangzhou University of Chinese Medicine, Guangzhou, 510405, China

${ }^{3}$ School of Pharmaceutical Sciences, Guangzhou University of Chinese Medicine, Guangzhou, 510405, China

Corresponding author :*Geng Li, lg@gzucm.edu.cn

Co-corresponding author : *Xiaohong Liu, rsclxh@gzucm.edu.cn

*Xiao Wang, xwang72@gzucm.edu.cn

First auther: ${ }^{+}$Qiong Zhang, 764378051@qq.com

Co-first author: ${ }^{+}$Xuemei Lin, 1301948005@qq.com

+Yanni Lai,865193910@qq.com

\begin{abstract}
Background: Zika fever is a mosquito-borne infection induced by Zika virus (ZIKV),effective drug and vaccine against ZIKV infection is still unavailable. Cinnamomi ramulus $(\mathrm{CR})$ is a traditional Chinese herb with a long history ,can stimulate secretion of sweat and relieve exterior syndrome. The aim of this research was to evaluate the effect of CR against ZIKV and uncover its mechanism of action by network pharmacology .
\end{abstract}

Methods: Cell viability assay, qRT-PCR assay and western blot assays were 
perfermed to evaluate anti-ZIKV activity in vitro. Survival rate, body weight were observed and viremia was detected in AG6 mice. Drug- target-disease networks, GO enrichment, and KEGG pathway analysis was established to clarify the therapeutic mechanism.

Results:In this study, we find that CR can alleviate cytopathic effect( CPE) after ZIKV infection. In the premixed administration mode, CR showed superior activity to inhibit viral RNA replication and protein expression in cells. Orally administered CR effectively protected AG6 mice infected with lethal doses of ZIKV, conferring $50 \%$ or $20 \%$ survival rate at a dosage of $900 \mathrm{mg} / \mathrm{ml}$ or $450 \mathrm{mg} / \mathrm{ml}$, reducing body weight loss, inhibiting viral RNA replication. Beta-sitosterol, DBP, $\alpha$-Longipinen, (-)-alpha-cedrene, ()-alpha-Longipinene may the main active compound and PTGS2, GABRA1, PTGS1, PTGES and CCR5 may the main targets. 176 biological processes (BP ) , 16 cell components(CC), 27 molecular functions(MF), and 37 pathways were significantly identified in GO enrichment and pathway analysis.

Conclusions: These results reveal that $\mathrm{CR}$ treatment of Zika fever is mainly related to PTGS1 and PTGS2 regulated prostaglandin(PG) release, metabolism, and inflammation response.

Keywords: Zika fever, Cinnamomi ramulus, Network pharmacology, Inflammation Background Zika fever is an acute illness induced by ZIKV, the patient has the mind fever $\left(37.8 \sim 38.5^{\circ} \mathrm{C}\right)$, rash in face and limbs, conjunctivitis, myalgia, arthritis and oedema in periarticular of the small joints of hands and feet. The symptoms are easy to confuse with Dengue, Chikungunya. Zika fever is usually self-limiting with most clinical manifestations resolving completely within 3 7days and the course of disease lasts less than a week, some patients have digestive system diseases such as nausea, vomiting, diarrhea, constipation, abdominal pain, and aphthous ulcers.And genitourinary system disease, such as haematuria, dysuria, perineal pain and haematospermia(1). The infection disease hasn't attracted attention until reported ZIKV infection can further cause severe congenital Zika syndrome in fetus and Guillain-Barre syndrome in adults has triggered widespread concern, Zika syndrome 
includes natural malformations, fetal death and microcephaly are caused by pregnant women infected with $\mathrm{ZIKV(2).} \mathrm{Guillain-Barre} \mathrm{syndrome} \mathrm{is} \mathrm{an} \mathrm{autoimmune} \mathrm{disease,}$ mainly affects the peripheral nervous system and can cause muscle paralysis and severe peripheral nerve disease,even death $(3,4)$. ZIKV is a positive-sense, single-stranded enveloped RNA virus, and dengue virus(DENV), West Nile virus (WNV), yellow fever virus (YFV), and Japanese encephalitis virus are belonging to the genus Flavivirus of the Flaviviridae family, has emerged in many countries and widespread in human population mainly by infected female Aedes aegypti and Aedes albopictus(5). Beside the major source of transmission mosquito bites, the virus can also be passed on through sexual contacts and blood transmissions, such as blood transfusion and share syringe(6). ZIKV is first isolated from three owl monkey in the Zika forest of Uganda in African by American scientists in 1947 and isolated from the blood of a Nigerian patient in 1956, even though the first human infection with ZIKV was reported(7). From the 1960 s to the 1980 s, only 15 ZIKV infection cases were scattered sporadically Africa and South Asia including Uganda, Egypt, Tanzania and Indonesia, Malaysia, Thailand, Vietnam District. In April, 2007, ZIKV broke out in Asia and Africa, firstly occurred in Micronesia United Endemic to Bang Yap Island, and caused about $70 \%$ of local residents were infected with ZIKV(8).In November 2015, ZIKV also triggered wide-scale outbreaks in Central and South America(9).On February 1, 2016, the World Health Organization (WHO) announced Fetal microcephaly and other related diseases caused by ZIKV have constituted "International Emergency Public Health events "and call on the international community to work together to respond(10). Currently, there are no vaccines and specific drugs to against ZIKV, the most effective way to prevent mosquito bites. Since WHO announced ZIKV as an international public health issue in 2016, ZIKV vaccines and drugs have become the hotpot of research. In the last two years,several candidate vaccines and anti-ZIKV drugs are under Phase I and Phase II clinical trials experimental evaluation (11-13), the absence of vaccines and drugs highlights an urgent need for new classes of anti-ZIKV agents to combat potential human ZIKV pandemics. Cinnamomi ramulus is the well-known Traditional Chinese medicinal 
herb from the dried branch and skin of the cinnamon family Cinnamomum cassia. From the perspective of traditional Chinese medicine(TCM), Cinnamomi ramulus can treat paralysis, dispel wind and cold, remove heat and sweat,etc(14). Modern research has found various pharmacological effects such as anti-viral(15), anti-inflammatory(16), anti-tumor(17), anti-bacterial(18) and neuroprotective activity, etc(19).

Based on the current research status of ZIKV and related to the experience of TCM against epidemic infection disease, we surprisely find that extracts from Cinnamomi Ramulus have obviously anti-ZIKV effects and never has seen reported. In this study,we examined the efficacy of CR against ZIKV in Vero-E6 cells and Hela cells based on the cytotoxicity assays. The CPE assay, qRT-PCR analysis and Western blot analysis methods were used to explored the anti-ZIKV activity in vitro. For the animal experiment, AG6 mice (ifnar $1^{-/}$ifngr $1^{--}$) is highly susceptible to ZIKV and thus the strain mice for the testing of CR antivirals activity in vivo(20). The survival rate of mice and viral RNA level expression in blood were observed in our study. TCM is a complex system of multiple components, multiple targets and multiple pathways. Network pharmacology is a new discipline emerging in recent years. Through network analysis, the interaction effects and potential molecular mechanisms among disease-target-drug active components are studied at the molecular level. In this study, network pharmacology was used to study the mechanism of CR for Zika fever and to provide a reference for the further research.

\section{Methods}

\section{Preparation of the plant and plant extract}

Cinnamomi ramulus was purchased from Guangxi Provinces in South China. The authenticity of these twigs was identified as the shape according to Chinese Pharmacopoeiaby Liangwen Yu professor of Guangzhou University of TCM. 50g Cinnamomi ramulus were pulverized and extracted with $300 \mathrm{ml}$ water for $30 \mathrm{~min}$ using a heating reflux method, the liquid was filtered and drug residues were extracted again with same methods. The liquid was combined and concentrated into $1 \mathrm{~g} / \mathrm{ml}$ (about 
$50 \mathrm{ml}$ ) using a rotary evaporator, the extracted was stored to $-20^{\circ} \mathrm{C}$ overnight and then dried with vacuum freeze dryer, the powder was diluted with culture medium before using during the experiment.

\section{Cells and viruses}

African Green Monkey Kidney cells (Vero-E6) and Henrietta Lacks cells (Hela) donated by the State Key Laboratory of Virology, Wuhan University, and Aedes albopictus cells (C6/36) purchased from the cell bank of Shanghai Chinese Academy of Sciences.The ZIKV (Zika virus/SZ01/2016/China) strain was provided by the CDC (Center for Disease Control and Prevention) of Guangdong Provincial and amplificated on $\mathrm{C} 6 / 36$ cells and stored at $-80^{\circ} \mathrm{C}$ until needed. The titer of ZIKV was determined in Vero-E6 by plaque assay.

\section{Cytotoxicity of CR by cell viability assay}

The cytotoxicity of $\mathrm{CR}$ was assessed by cell viability assay with MTT (3-(4,5-dimethylthiazol-2-yl)-2,5-diphenyl tetrazolium bromide working solution, 5 $\mathrm{mg} / \mathrm{mL}$ ). Vero-E6 and Hela cells were seeded at $2 \times 10^{4}$ cells /well and incubated for $18 \mathrm{~h}$ at $37^{\circ} \mathrm{C}$ in a humidifed incubator with $5 \% \mathrm{CO}_{2}$, cells were inoculated with various concentrations containing 2\% Fetal Bovine Serum (FBS) Dulbecco's Modified Eagle medium (DMEM) of $\mathrm{CR}$ for $48 \mathrm{~h}$ at $37^{\circ} \mathrm{C}, 5 \% \mathrm{CO}_{2} .20 \mu \mathrm{L}$ of the 3-(4,5-dimethylthiazol-2-yl)-2,5-diphenyl tetrazolium bromide working solution (MTT,5 mg/mL) was added and incubated for $4 \mathrm{~h} .150 \mu \mathrm{L}$ of dimethyl sulfoxide (DMSO) was added to dissolve the formazan crystals and the absorbance was measured with $490 \mathrm{~nm}$ wavelength by multimode microplate reader (PerkinElmer).The cell viability rate of $\mathrm{CR}$ was calculated using the following formula:

Cell viability rate $(\%)=[($ mean optical dentisity of test-mean optical density of density of black controls)/(mean optical density of cell controls-mean optical density of black controls) $] \times 100 \%$

\section{Antiviral activities of CR under different modes of administration}

Vero-E6 cells were plated at $1 \times 10^{4}$ cells/well in a 96 -well plate incubated at 
$37^{\circ} \mathrm{C}$ in $5 \% \mathrm{CO}_{2}$ for $18 \mathrm{~h}$. Permixed administration, simultaneous administration and therapeutic administration modes were performed to determine the mode of action of CR. The cells were observed CPE by Microscope and $20 \mu \mathrm{L}$ MTT was added and incubated for $4 \mathrm{~h} .150 \mu \mathrm{L}$ of DMSO was added to dissolve the formazan crystals and the absorbance was measured with $490 \mathrm{~nm}$ wavelength by multimode microplate reader. The cell viability rate of $\mathrm{CR}$ was calculated using the following formula :

Cell viability $(\%)=[($ mean optical density of test-mean optical density of virus controls $) /($ mean optical density of cell controls-mean optical density of virus controls)] $\times 100 \%$

\section{Antiviral activities of CR by qRT-PCR analysis}

Vero-E6 cells and Hela cells were plated at $1 \times 10_{5}$ cells/well in a 12 -well plate incubated at $37^{\circ} \mathrm{C}$ in $5 \% \mathrm{CO}_{2}$ for $18 \mathrm{~h}$. Permixed administration, simultaneous administration and therapeutic administration modes were performed. The cells were collected and total RNA was extracted using the Ultrapure RNA kit(Co Win Biotech, Beijing,China), cDNA was obtained using the M-MLV Reverse Transcriptase kit (Promega,Madison,WI,United States) and the amplification conditions: at 1 cycle $95{ }^{\circ} \mathrm{C}$ for $3 \mathrm{~min} ; \quad 39$ cycles at $95^{\circ} \mathrm{C}$ for $10 \mathrm{~s} ; 60^{\circ} \mathrm{C}$ or $10 \mathrm{~s}$, and $72{ }^{\circ} \mathrm{C}$ for $20 \mathrm{~s}$; and 1cycles at $95{ }^{\circ} \mathrm{C}$ for 10s. The specific primer for ZIKV:Forward primer:GGTCAGCGTCCTCTCTAATAAACG,Reverse

primer:GCACCCTAGTGTCCACTTTTTCC; GAPDH:Forward primer:AAGGCTGTGGGCAAGG, Reverse primer:TGGAGGAGTGGGTGTCG

The inhibition rate was measured using CFX connect quantitative Real-Time PCR instrument.

\section{Antiviral activities of CR by Western blot analysis}

Vero-E6 cells and Hela were plated at $2 \times 10^{5}$ cells/well in a 6 -well plate incubated at $37^{\circ} \mathrm{C}$ in $5 \% \mathrm{CO}_{2}$ for $18 \mathrm{~h}$. Permixed administration, simultaneous administration and therapeutic administration modes were performed and cells were collected and lysed with lysis buffer, total protein was collected by centrifugation at $10,000 \times \mathrm{g}$ for $10 \mathrm{~min}$ at $4{ }^{\circ} \mathrm{C}$, the denatured protein was separated by $10 \%$ sodium dodecyl 
sulfate-polyacrylamide gel electrophoresis, transferred onto a polyvinylidene difluoride membrane, blocked with 5\% skim milk, and sequentially incubated with anti-NS5, anti-NS3, anti-E, anti-prM and GAPDH primary antibodies (GeneTex, San Antonio, USA), followed by incubation with a secondary antibody. The tegrated bands were analyzed using FluorChem $\mathrm{R}$ multifunctional imaging machine(protein simple, USA).

\section{Antiviral activities of CR by Survival rate of mice and viral RNA replication}

AG6 mice were divided into 4 groups $(n=6)$ used for the experiment, provided by the Laboratory Animal Services Center, Guangzhou University of Chinese Medicine, female aged 5 6 weeks, housed in individually ventilated cages. The temperature was maintained at $22 \pm 2^{\circ} \mathrm{C}$ and humidity at $50 \pm 10 \%, 12 \mathrm{~h} / 12 \mathrm{~h}$ light/dark cycles. The mice were challenged by injecting with $3.3 \times 10^{6}$ pfu of ZIKV or $0.9 \%$ sterile saline $50 \mu 1$. CR was orally administered with $900 \mathrm{mg} / \mathrm{kg}$ and $450 \mathrm{mg} / \mathrm{kg}$, the orbital blood was collected on day 3 and day 5 post-infectionon and the qRT-PCR assay as previously described (28). The mice were monitored the weight, number and time of death daily until day 15. The experiment was carried out in animal class II biosafety safety cabinets.

\section{Collection targets of candidate compounds and Zika fever}

The compounds of CR were collected by the TCMSP database (TCMSP, http://tcmspw.com/tcmsp.php). Considering that Zika fever is related with bloodbrain barrier, so ADME (absorption, distribution, metabolism, excretion) parameters selected $\mathrm{OB}$ (oral bioavailability) $\geq 30 \%$, DL(drug-likeness) $\geq 0.1, \mathrm{BBB}(\mathrm{Blood}$ - brain barrier) $\geq 0.3$ as the filter conditions of the active ingredients. In addition, Some compounds were classified into the candidate compound even not meet the screening criteria, but were essential pharmacological ingredients and with significant antiviral effects on the human body. The potential targets of candidate compounds were searched from the TCMSP database and Swiss Target Prediction (http://www.swisstargetprediction.ch/), the official gene symbols were acquired and listed using the UniProt database (http://www.uniprot.org). The targets related to Zika 
fever were provided by Online Mendelian Inheritance in Man (OMIM,https://www.omim.org/).

\section{Construction Zika fever -related protein-protein interaction (PPI) network and}

\section{Drug- Target-Disease Network}

The targets of compounds and Zika fever disease were input Draw Venn Diagram website (http://bioinformatics.psb.ugent.be/webtools/Venn/), the overlapping targets were showed by the Venn diagram .PPI network was constructed to explore the direct and indirect interaction relationship among the targets by STRING database (https://string-db.org/). Cytoscape3.7.2 software was used to construct the drug-overlapping target-disease interaction network. The topological properties of networks were evaluated by the Cytoscape 3.7.2 sofeware.

\section{GO enrichment and KEGG pathway enrichment}

In order to elucidate the role of targets that interact with the candidate compound of $\mathrm{CR}$ in gene function and signaling pathway, the overlapping compound targets for GO enrichmentand KEGG pathway enrichment analysis using STRING datebase. The potential targets involved in the $\mathrm{BP}, \mathrm{CC}, \mathrm{MF}$, and the pathways were also described.

\section{Statistical analysis}

The results were analyzed by one-way analysis of variance (ANOVA) or Dunnett's T3 when the data were not normally distributed.The date showed as the mean \pm standard, P-values of less than or equal to 0.05 were considered statistically significant.

\section{Result}

\section{CR can represses ZIKV infection in Vero-E6 cells}

The $50 \%$ Cytotoxic Concentration of $\mathrm{CR}\left(\mathrm{CC}_{50}\right)$ was $>4500 \mu \mathrm{g} / \mathrm{ml}$ in Vero-E6 cells and $3396 \mu \mathrm{g} / \mathrm{ml}$ in Hela cells(Fig.1a and 1b). CR displayed different anti-ZIKV activities under different modes of medication. CPE was monitored by direct microscopic observations(Fig.1c). Compared with the virus control, far less CPE than in CR treated groups. The cell viability rate was deterninated by using the MTT, CR 
displayed a clear anti-ZIKV activity in Vero-E6 cells. Compared with other medication modes, the premixed administration modes showed a superior antiviral activity, the inhibition rate was up to $100 \%$ at the concentration of $100 \sim 600 \mu \mathrm{g} / \mathrm{ml}$ (Fig.1d-1f).

\section{CR can inhibit ZIKV replication in Vero-E6 cells and Hela cells}

Based on the result of the CPE reduction assay, qRT-PCR assay was carried out to determine the level of viral RNA in Vero-E6 cells and Hela cells at different medication modes. The result showed that CR can inhibit viral RNA synthesis, the premixed medicine therapy mode was the better mode than other modes and the $50 \%$ effective inhibitory concentrations $\left(\mathrm{EC}_{50}\right)$ was $<25 \mu \mathrm{g} / \mathrm{ml}$ in Vero-E6 cells(Fig.2a-2c) and $74 \pm 1.50 \mu \mathrm{g} / \mathrm{ml}$ in Hela cells(Fig.2d-2f). The specific $\mathrm{EC}_{50}$ and selection index(SI) of CR was displayed in the (Table1).

\section{CR can inhibit the expression of ZIKV protein in Vero-E6 cells}

In order to further explored the antiviral activity of $\mathrm{CR}$, the Western blot assay was performed under the different medication modes. The experiment result showed that CR can almost completely inhibit the expression of NS5, NS3, prM and E protein of ZIKV with the concentration of $25 \sim 500 \mu \mathrm{g} / \mathrm{ml}$ at the premixed administration modes(Fig.2g). However, the simultaneous and therapeutic administration modes didn't disply a good inhibition activity of ZIKV protein as the premixed therapy(Fig.2e and 2f). Under the therapeutic administration mode, CR can't inhibit the expression of proteins with the concentration $25 \sim 300 \mu \mathrm{g} / \mathrm{ml}$, but can inbit the NS5 and $\mathrm{E}$ protein expression at the $500 \mu \mathrm{g} / \mathrm{ml}$ and only can inhibit E protein expression at the $400 \mu \mathrm{g} / \mathrm{ml}$. In the summary, the result indicated the premixed administration modes playing a significant role in the antiviral process.

\section{CR can reduces the mortality of mice infected $\mathrm{ZIKV}$}

AG6 mice were used to test the efficiency of CR and the normal group mice were survived healthily and no death during the 15 observation. The ZIKV-infected mice presented signs of illness symptoms, inactivity,hind limb paralysis, weight loss and lack of appetite on the fourth day, the body weight of all the survival mice were gained again on the twelves days(Fig.3b). The model group mice were began to die at 
days 7 post-infection and all the mice were dead on ninth days, loss $30 \%$ body weight was considered dead. CR treated mice were obviously protected and the survival rate were $50 \%$ ( $900 \mathrm{mg} / \mathrm{kg}$ ) and 30\% ( $450 \mathrm{mg} / \mathrm{kg}$ ), respectively(Fig.3a). The viral RNA level was measured by qRT-PCR alalysis on day 3 and day 5 post-infection. Compared with ZIKV group, the mice of viral RNA copies all reduced after the dose of $900 \mathrm{mg} / \mathrm{kg}$ and $450 \mathrm{mg} / \mathrm{kg}$ administered, however, the dose of $450 \mathrm{mg} / \mathrm{kg}$ had no difference with ZIKV model group on the day 3 post-infection but had difference on the day 5 post-infection. Comparison of ZIKV infected mice on day 3 and ZIKV infected on day 5, the viremia has statistically significant, the viral RNA level in blood was higher on day 3 than day 5 , the reason may be virus was through the blood conveyed to the tissues (Fig.3c) .On the whole, the result presented that CR can inhibit ZIKV in vivo by the inhibit the viral RNA replication.

\section{Screening for Active Compounds and fishing targets}

Considered CR mainly take effects by oral administration, OB, DL and BBB parameters were used to screen the active compounds of Zika fever. 664 targets were identified from 24 compounds and 5 mainly pharmacological ingredients, 557 known therapeutic targets were obtained from the disease Zika fever. 20 targets in CR were associated with Zika Fever (Fig.4a), we matched 24 compounds with the the overlapping targets.

\section{PPI network and Drug- Target-Disease Network analysis}

20 nodes and 30 edges constructed PPI network, the multiple therapeutic targets were PTGER3, NLRP3, KCNH2, PTGES, TNFRSF1A, PTGS2, ITK, GABRA1, HTR1A, G6PD, GABRB3, PLG, DYRK1A, JAK1, GABRG2, CCR5, IL1B, PTGS1, CSF1R, SCN5A. PPI network analysis showed that enrichment p-value were 5.28e-11 and average node degree were 3, the color of line indicates the type of interaction evidence (Fig.4b). 48 nodes and 136 edges constructed Drug-Target -Disease Network (Fig.4c). The network was evaluated by network centralization and network heterogeneity, the valus were 0.429 and 0.888 respectively, the result indicated that some nodes are more concentrated in the network than others and means that the 
compound target space has a tendency for certain compounds and targets. We can know PTGS2(degree=20), GABRB3(degree=15), PTGS1(degree=14) , PTGES(degree=7) and CCR5(degree=7) were probably the main targets, and Beta-sitosterol $\quad($ degree $=8), \quad \operatorname{DBP}($ degree $=8), \quad \alpha$-Longipinene $($ degree $=7)$, $(-)$-alpha-cedrene(degree $=7)$ and ()-alpha-Longipinene $($ degree=6) were main active compound to take antiviral effects and treat Zika fever (Table2 and Table 3).

\section{GO enrichment and KEGG enrichment analysis}

To analyze the functional characteristics of 20 identified targets, 219 terms were found according to $\mathrm{P}<0.05$ by STRING. 176 enriched terms in BP categories, 16 enriched terms in $\mathrm{CC}$ categories and 27 enriched terms in MF categories. The top 5 significantly enriched terms in BP, MF, and CC categories (Fig.5a). In the BP categories, the target proteins were mainly involved in defense respons, inflammatory response, prostaglandin metabolic process, positive regulation of fever generation, cellular response to chemical stimulus. In the $\mathrm{CC}$ categories, the target proteins were mainly involved in plasma membrane part, integral component of plasma membrane , membrane part, cell surface, side of membrane. In the CC categories, the target proteins were mainly involved in prostaglandin-endoperoxide synthase activit, protein tyrosine kinase activity, non-membrane spanning protein tyrosine kinase activity, GABA-gated chloride ion channel activity, transmembrane signaling receptor activity. The GO enrichment analysis results showed that the active ingredients of CR could regulate prostaglandin and kinase in cell membrane and plasma membrane in the process of inflammation, so as to exert anti-inflammatory and antiviral activity.

In order to further clarify the relationship between target proteins and the pathways, KEGG enrichment was performed 37 term pathways were found according to $\mathrm{P}<0.05$ by STRING, the top 20 significantly enriched pathways were mainly involved in Influenza A, Neuroactive ligand-receptor interaction, Osteoclast differentiation Serotonergic synapse, Necroptosis, Regulation of lipolysis in adipocytes, Arachidonic acid metabolism, Kaposi's sarcoma-associated herpesvirus infection, Leishmaniasis, Cytokine-cytokine receptor interaction, NF-kappa B 
signaling pathway, etc. (Fig.5c)

\section{Discussion and Conclusion}

At present study and clinical diagnosis reported, Zika fever is usually asymptomatic or mild in adults by ZIKV infection, but ZIKV can quickly resolve blood viremia and persist for months in saliva, urine, semen, breast milk and the central nervous system and further developed Zika syndrome and Guillain-Barre syndrome $(21,22)$. Therefore, outbreaks and epidemics of ZIKV are caused a huge threats to human health, especially pregnant women and newborns. The absence licensed drugs highlight the importance to discover and develop more novel antiviral agents.

As previously reported, Vero-E6 cell strain is easily infected by ZIKV (23) and Hela cell strain is also susceptible to $\mathrm{ZIKV}(24)$. For the reason that we choose Vero-E6 cell strain and Hela cell strain for our experimental research. First of all, we found that CR had a limited cytotoxicity by detecting the cell viability using MTT in Fig. 1a and the $\mathrm{CC}_{50}$ values were $>4500 \mu \mathrm{g} / \mathrm{ml}$ in Vero-E6 cells and $3396 \mu \mathrm{g} / \mathrm{ml}$ in Hela cells. Because ZIKV can produce a rapid and visible CPE by observing cell death in vitro, we carried out the CPE assay to explore the antiviral activity of herb extracts. surprisingly, CR can effectively reduce cytopathy and protect the cell from ZIKV, the results were showen in Fig.1b. Different drugs have different antiviral action modes due to the stage of viral replication, premixed, simultaneous and therapeutic admonostration modes were performed according to the previously described(25). The result showed that $\mathrm{CR}$ had a significant effect at premixed administration mode to inhibit the replication of ZIKV in Vero-E6 cells and Hela cells, the $\mathrm{EC}_{50}$ values were $45.75 \mu \mathrm{g} / \mathrm{ml}$ in Vero-E6 cells and $74.02 \mu \mathrm{g} / \mathrm{ml}$ in Hela cells, the Select Index(SI) values were more than 98.36 and 45.88, the data was showed in Fig.1c and Table 1. NS5, NS3, E and prM protein were the main proteins of ZIKV and their expression levels are essential for ZIKV to reflect the extent of viral protein synthesis. Compared with the other treatment modes, the premixed administration mode showed a better inhibitory effect in Fig.2b. The result is completely consistent with qRT-PCR assays 
and showed obvious inhibition in viral protein synthesis. Taken together, our results verified that $\mathrm{CR}$ has superior against ZIKV activity in vitro.

AG6 mice were can be effectively used as murine models for Zika virus research. In order to further confirm whether CR has an inhibitory influence on ZIKV-induced mouse model, AG6 mice were used in our study, the result showed CR exhibited a significant mortality protection of mice against ZIKV infection, the infected mice were trated with $\mathrm{CR}$ showed a obviously delay in mortality and protected the body weight loss in Fig. $3 \mathrm{a}$ and $3 \mathrm{~b}$, the survival rate is up to $50 \%$ when the mice were treated at the high dose of $900 \mathrm{mg} / \mathrm{kg}$ and $30 \%$ survival rate at the low dose of $450 \mathrm{mg} / \mathrm{kg}$. In addition, the viremia was monitored at day 3 and day 5 post-infection, the date showed that $\mathrm{CR}$ can reduce the viremia at 5 post-infection in Fig. $3 \mathrm{c}(26)$. TCM is a great treasure and has a long history in China, multiple ingredients, multiple targets and multiple pathways were the features of TCM to play pharmacological effect. Strongly active against ZIKV was shown in our work and futher studies to explore the target and mechanism of anti-ZIKV from network pharmacological analysis, we found Beta-sitosterol, DBP, $\alpha$-Longipinene, (-)-alpha-cedrene, ()-alpha-Longipinene may the main active compound and PTGS2, GABRA1, PTGS1, PTGES and CCR5 may the main targets. Zika fever can induce upper respiratory tract infection and is similar to influenza A infection, and futher develope Severe limb paralysis and microcephaly caused by condition worsening. It has been reported that PTGS1 can regulate lipopolysaccharide-induced inflammation and brain damage(27) and PTGS2 can promote the occurrence of inflammatory neuropsychiatric disorders(28). PTGS1 and PTGS2 oxidize arachidonic acid to PG and regulate the body's homeostasis and inflammation during PG synthesis(29). PG were widely distributed in the central nervous system and can regulate the release and activity of neurotransmitters, has a regulatory effect on autonomic nerve media and regulate brain growth and physical activity, enhance the sensitivity of pain receptors to pain-causing substances and their pain-causing effects(30). The release of PG can also amplify inflammatory pain and induce inflammation-redness, swelling, heat and 
pain(31). Fever is a common symptom of infectious and inflammatory disease. It is well-established that prostaglandin E2 is the final mediator of fever, which by binding to its EP3 receptor subtype in the preoptic hypothalamus initiates thermogenesis(32).The GO enrichment and KEGG enrichment analysis result showed that $\mathrm{CR}$ relieved Zika fever and condition deterioration by regulating the expression of PTGS2 and PTGS1 to further inhibit PG release and regulate metabolism to reduce inflammation response.

\section{Conclusion}

In conclusion, Zika fever, as an infectious diseases have strong ability to spread in endemic areas and cause a serious harm to pregnant women and fetus. Our findings demonstrated that CR shows potentially effective against ZIKV in vitro and vivo, the data provides reference basis for the prevention and treatment of Zika fever. Beta-sitosterol, DBP, $\alpha$-Longipinen, (-)-alpha-cedrene, ()-alpha-Longipinene may the main active compound of CR to play an antiviral role and PTGS2, GABRA1, PTGS1, PTGES and CCR5 may the main targets related to Zika fever induced by ZIKV. The mechanism of CR treatment of Zika fever may be related to PTGS1 and PTGS2 mediated PG release, metabolism and inflammation response, but the detailed verification about active compounds and potential targets experiments need further research and performed.

\section{Abbreviations}

ZIKV: Zika virus; CR:Cinnamomi ramulus; CPE: cytopathic effect; BP: biological processes; CC: cell components; MF: molecular functions ; PG: prostaglandin; DENV: dengue virus; WNV: West Nile virus ; YFV: yellow fever virus ; WHO: World Health Organization; TCM:traditional Chinese medicine; Vero-E6: African Green Monkey Kidney cells; Hela: Henrietta Lacks cells ; C6/36: Aedes albopictus cells; CDC: Center for Disease Control and Prevention; MTT: 3-(4,5-dimethylthiazol-2-yl)-2,5-diphenyl tetrazolium bromide; FBS: Fetal Bovine Serum; DMEM: Dulbecco's Modified Eagle medium; DMSO: dimethyl sulfoxide; ADME: absorption,distribution,metabolism,excretion ; $\mathrm{CC}_{50}$ : The 50\% Cytotoxic 
Concentration; $\mathrm{EC}_{50}$ : The 50\% effective inhibitory concentrations ; OB: oral bioavailability; DL: drug-likeness; BBB: blood- brain barrier; SI: Select Index.

\section{Acknowledgement}

This work was supported by ABSL-2 Laboratory of Guangzhou University of Traditional Chinese Medicine. We sincerely thank professor Jianguo Wu (Wuhan University) for providing the Zika virus and professor Jincun Zhao (State Key Laboratory Respiratory Disease, Guangzhou medical university) for providing transgenic mice.

\section{Authors'contributions}

Geng Li, Xiaohong Liu and Xiao Wang organized and supervised the study. Qiong Zhang, Xuemei Lin, Yanni Lai, Jiangtao Xu and Yuting Chen drafted the manuscript and prepared tables and fgures. Qiong Zhang and Xuemei Lin contributed to revisions of the manuscript. All authors read and approved the fnal manuscript.

\section{Funding}

National Nature Science Foundation of China (81803813, 81973814), The Open Project of State Key Laboratory of Natural Medicines (SKLNMKF201906), Science \& Technology Planning Project of Guangdong Province Office of Education(2019KYTD102, 2020KZDZX1060), Guangdong province key areas R \& D plan "Lingnan Chinese medicine modernization" （192019082450200013）

\section{Availability of data and materials}

All data generated or analysed during this study are included in this published article.

\section{Ethics approval and consent to participate}

Not applicable.

\section{Consent for publication}

Not applicable.

\section{Competing interests}


The authors declare that they have no competing interests.

\section{Author details}

${ }^{1}$ Laboratory Animal Center, Guangzhou University of Chinese Medicine, Guangzhou, 510405, China

${ }^{2}$ The First Clinical Medical College, Guangzhou University of Chinese Medicine, Guangzhou, 510405, China

${ }^{3}$ School of Pharmaceutical Sciences, Guangzhou University of Chinese Medicine, Guangzhou, 510405, China

\section{References}

1. Chan JF, Choi GK, Yip CC, Cheng VC, Yuen KY. Zika fever and congenital Zika syndrome: An unexpected emerging arboviral disease. J Infect. [Journal Article; Research Support, Non-U.S. Gov't; Review]. 2016 2016-05-01;72(5):507-24.

2. Costa F, Sarno M, Khouri R, de Paula Freitas B, Siqueira I, Ribeiro GS, et al. Emergence of Congenital Zika Syndrome: Viewpoint From the Front Lines. ANN INTERN MED. 2016 2016-01-01;164(10):689-91.

3. Leonhard SE, Bresani-Salvi CC, Lyra Batista JD, Cunha S, Jacobs BC, Brito Ferreira ML, et al. Guillain-Barré syndrome related to Zika virus infection: A systematic review and meta-analysis of the clinical and electrophysiological phenotype. PLOS NEGLECT TROP D. 2020 2020-04-27;14(4):e8264.

4. Pimentel R, Jubithana B, Hernandez F, Montoya R, Lara B, Melchor A, et al. Zika Virus and the Guillain - Barré Syndrome - Case Series from Seven Countries. The New England Journal of Medicine. 2016 2016-01-01;375(16):1598-601.

5. Wang Z, Wang P, An J. Zika virus and Zika fever. VIROL SIN. [Journal Article; Review]. 2016 2016-04-01;31(2):103-9.

6. Gregory CJ, Oduyebo T, Brault AC, Brooks JT, Chung K, Hills S, et al. Modes of Transmission of Zika Virus. The Journal of Infectious Diseases. 2017 2017-12-16;216(suppl_10):S875-83.

7. Karkhah A, Nouri HR, Javanian M, Koppolu V, Masrour-Roudsari J, Kazemi S, et al. Zika virus: epidemiology, clinical aspects, diagnosis, and control of infection. 
EUR J CLIN MICROBIOL. 2018;37(11):2035-43.

8. Baud D, Gubler DJ, Schaub B, Lanteri MC, Musso D. An update on Zika virus infection. The Lancet. 2017;390(10107):2099-109.

9. Ladhani SN, O'Connor C, Kirkbride H, Brooks T, Morgan D. Outbreak of Zika virus disease in the Americas and the association with microcephaly, congenital malformations and Guillain - Barré syndrome. ARCH DIS CHILD. 2016 2016-01-01;101(7):600-2.

10. Imperato PJ. The Convergence of a Virus, Mosquitoes, and Human Travel in Globalizing the Zika Epidemic. J COMMUN HEALTH. 2016;41(3):674-9.

11. Wilder-Smith A, Vannice K, Durbin A, Hombach J, Thomas SJ, Thevarjan I, et al. Zika vaccines and therapeutics: landscape analysis and challenges ahead. BMC MED. 2018;16(1).

12. Boldescu V, Behnam MAM, Vasilakis N, Klein CD. Broad-spectrum agents for flaviviral infections: dengue, Zika and beyond. NAT REV DRUG DISCOV. 2017;16(8):565-86.

13. Gaudinski MR, Houser KV, Morabito KM, Hu Z, Yamshchikov G, Rothwell RS, et al. Safety, tolerability, and immunogenicity of two Zika virus DNA vaccine candidates in healthy adults: randomised, open-label, phase 1 clinical trials. The Lancet. 2018;391(10120):552-62.

14. Liu J, Zhang Q, Li RL, Wei SJ, Huang CY, Gao YX, et al. The traditional uses, phytochemistry, pharmacology and toxicology of Cinnamomi ramulus: a review. J PHARM PHARMACOL. 2020 2020-01-01;72(3):319-42.

15. Fatima M, Zaidi NS, Amraiz D, Afzal F. In Vitro Antiviral Activity of Cinnamomum cassia and Its Nanoparticles Against H7N3 Influenza A Virus. J MICROBIOL BIOTECHN. 2016 2016-01-28;26(1):151-9.

16. Hong J, Yang G, Kim YB, Eom SH, Lew J, Kang H. Anti-inflammatory activity of cinnamon water extract in vivo and in vitro LPS-induced models. BMC COMPLEM ALTERN M. 2012 2012-01-01;12(1):237.

17. Shin SH, Lee SR, Lee E, Kim KH, Byun S. Caffeic Acid Phenethyl Ester from the Twigs ofCinnamomum cassia Inhibits Malignant Cell Transformation by Inducing 
c-Fos Degradation. J NAT PROD. 2017 2017-07-28;80(7):2124-30.

18. El Atki Y, Aouam I, El Kamari F, Taroq A, Nayme K, Timinouni M, et al. Antibacterial activity of cinnamon essential oils and their synergistic potential with antibiotics. Journal of Advanced Pharmaceutical Technology \& Research. 2019;10(2):63.

19. Liu X, Fu J, Yao X, Yang J, Liu L, Xie T, et al. Phenolic Constituents Isolated from the Twigs ofCinnamomum cassia and Their Potential Neuroprotective Effects. J NAT PROD. 2018 2018-06-22;81(6):1333-42.

20. Sun P, Nie K, Zhu Y, Liu Y, Wu P, Liu Z, et al. A mosquito salivary protein promotes flavivirus transmission by activation of autophagy. NAT COMMUN. 2020;11(1).

21. Tonnerre P, Melgaço JG, Torres-Cornejo A, Pinto MA, Yue C, Blümel J, et al. Evolution of the innate and adaptive immune response in women with acute Zika virus infection. NAT MICROBIOL. 2020;5(1):76-83.

22. Bonaldo MC, Ribeiro IP, Lima NS, Dos Santos AAC, Menezes LSR, Da Cruz SOD, et al. Isolation of Infective Zika Virus from Urine and Saliva of Patients in Brazil. PLOS NEGLECT TROP D. 2016 2016-06-24;10(6):e4816.

23. Vicenti I, Boccuto A, Giannini A, Dragoni F, Saladini F, Zazzi M. Comparative analysis of different cell systems for Zika virus (ZIKV) propagation and evaluation of anti-ZIKV compounds in vitro. VIRUS RES. 2018;244:64-70.

24. Ramos Da Silva S, Cheng F, Huang IC, Jung JU, Gao SJ. Efficiencies and kinetics of infection in different cell types/lines by African and Asian strains of Zika virus. J MED VIROL. 2018;91(2):179-89.

25. Lai Z, Ho Y, Lu J. Cephalotaxine inhibits Zika infection by impeding viral replication and stability. BIOCHEM BIOPH RES CO. 2020;522(4):1052-8.

26. Peng M, Watanabe S, Chan KWK, He Q, Zhao Y, Zhang Z, et al. Luteolin restricts dengue virus replication through inhibition of the proprotein convertase furin. ANTIVIR RES. 2017;143:176-85.

27. Choi SH, Langenbach R, Bosetti F. Genetic deletion or pharmacological 
inhibition of cyclooxygenas - 1 attenuate lipopolysaccharide - induced inflammatory response and brain injury. The FASEB Journal. 2008;22(5):1491-501. 28. Westwell-Roper C, Stewart SE. Commentary: Neurobiology and Therapeutic Potential of Cyclooxygenase-2 (COX-2) Inhibitors for Inflammation in Neuropsychiatric Disorders. FRONT PSYCHIATRY. 2020 2020-04-22;11.

29. Yagami T, Koma H, Yamamoto Y. Pathophysiological Roles of Cyclooxygenases and Prostaglandins in the Central Nervous System. MOL NEUROBIOL. 2016;53(7):4754-71.

30. Anton RF, Randall CL. Central Nervous System Prostaglandins and Ethanol. Alcoholism: Clinical and Experimental Research. 1987 1987-01-01;11(1):10-8.

31. Ricciotti E, FitzGerald GA. Prostaglandins and Inflammation. Arteriosclerosis, Thrombosis, and Vascular Biology. 2011 2011-01-01;31(5):986-1000.

32. Blomqvist A, Engblom D. Neural Mechanisms of Inflammation-Induced Fever. The Neuroscientist. 2017;24(4):381-99. 


\section{Figure Legends}

Fig.1 Cytotoxicity of CR and cell viability rate were evaluated by cell viability assay. (a )The $\mathrm{CC}_{50}$ of $\mathrm{CR}$ were determined by cell viability assay in Vero-E6 cells. (b ) The $\mathrm{CC}_{50}$ of $\mathrm{CR}$ were determined by cell viability assay in Hela cells. (c ) CPE reduction assay examining the effects of CR on ZIKV-infected Vero-E6 cells compared with the control and model. Different modes of administration, the cell survival rates of CR on ZIKV were determined by cell viability assay at $48 \mathrm{~h}$ post-infection. (d) Premixed administration: Drugs and virus were mixed, incubated at $4^{\circ} \mathrm{C}$ for $2 \mathrm{~h}$, and then added to the cell culture mixture. (e) Simultaneous administrations: Drugs and virus were mixed and immediately added to the cell culture. (f) Therapeutic administration:Drugs were added at $2 \mathrm{hpi}$ by the virus to MDCK cells. $n=3$, each concentration conducted in tripicate.

Fig.2 Antiviral activities of CR by qRT-PCR analysis and Western blot assays. (a-c) Inhibitory effects of various concentrations of $\mathrm{CR}$ administered in three infection protocols on ZIKV-infected Vero-E6 cells by qRT-PCR assay, $n=3$, each concentration conducted in tripicate. (d-f) Inhibitory effects of various concentrations of CR administered in three infection protocols on ZIKV-infected Hela cells by qRT-PCR assay. $n=3$, each concentration conducted in tripicate. (g-j) Level ZIKV proteins expression effects of various concentrations of $\mathrm{CR}$ administered in three infection protocols on ZIKV-infected Hela cells by Western-blot .

Fig.3 Therapeutic effect of CR on ZIKV in AG6 mice. (a) Mice were infected with $3.3 \times 10^{6} \mathrm{pfu}$ of $\mathrm{ZIKV}$, the survival rate and survival days were monitored until 15 days by receiveing oral administration of the saline or CR $(900 \mathrm{mg} / \mathrm{ml}, 450 \mathrm{mg} / \mathrm{ml})$. (b) Mice were infected with $3.3 \times 10^{6}$ pfu of $\mathrm{ZIKV}$, the body weight lossing were monitored until 15 days by receiveing oral administration of the saline or CR (900 $\mathrm{mg} / \mathrm{ml}, 450 \mathrm{mg} / \mathrm{ml}$ ). (c) Blood samples were collected on day 3 and day 5 p.i and subjected to qRT-PCR and detected viral RNA replication level. $n=6$, date are 
represented as the mean \pm S.D. $* P<0.05 ; * * P<0.01 ; * * * P<0.001$.

Fig.4 Screening of active compounds and potential targets. (a) The 20 matching targets of the related targets in $\mathrm{CR}$ on Zikv fever. (b) The PPI network was constructed by 20 nodes and 30 edges in the String database. (c) The DrugTarget-Disease network that consists of 48 nodes and 136 edges. yellow nodes denote the $\mathrm{CR}$, red nodes denote the disease-Zika fever,orange nodes denote the active ingredients and blue nodes denote the potential targets,respectively. Node size is relative to the degree, and the edges demonstrate the interaction between nodes.

Fig.5 GO enrichment and KEGG enrichment analysis of overlapping targets. (a) Top5 significantly enriched terms are shown in the biological process, cell component, and molecular function from STRING database. (b) Percentage of three items are shown by GO enrichment analysis. (c) The top 20 remarkably enriched pathways are shown. 
a

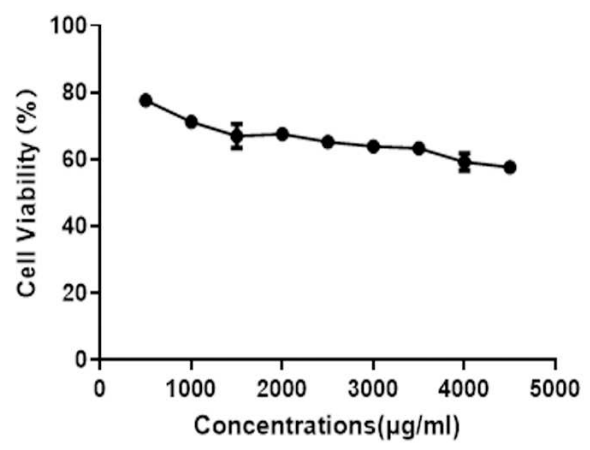

C

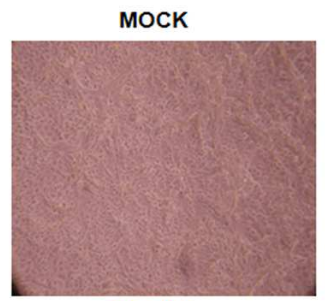

$50 \mu \mathrm{g} / \mathrm{ml}$

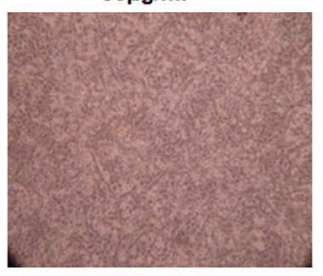

$300 \mu \mathrm{g} / \mathrm{ml}$
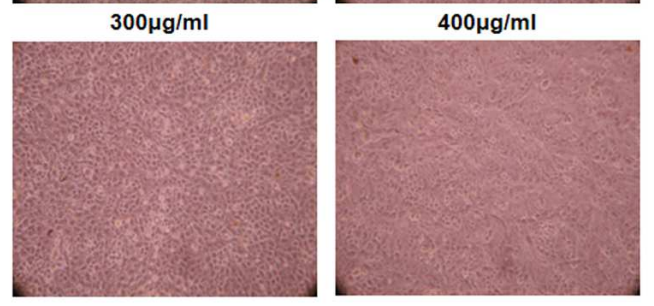

b

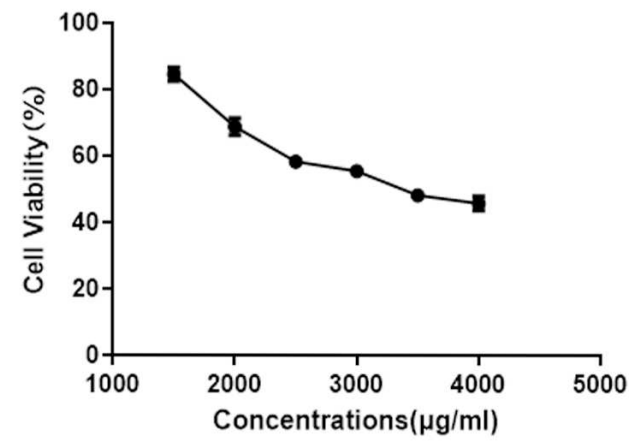

d

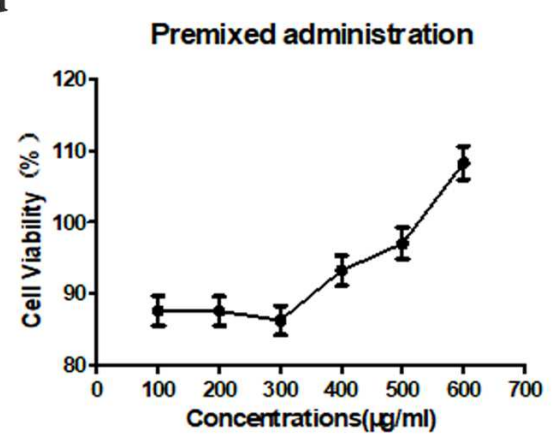

$\mathbf{e}$

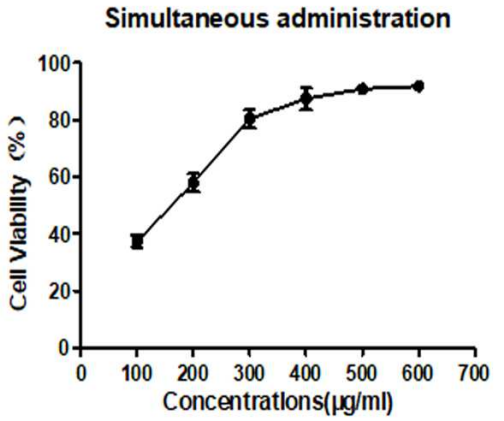

f

Therapeutic administration

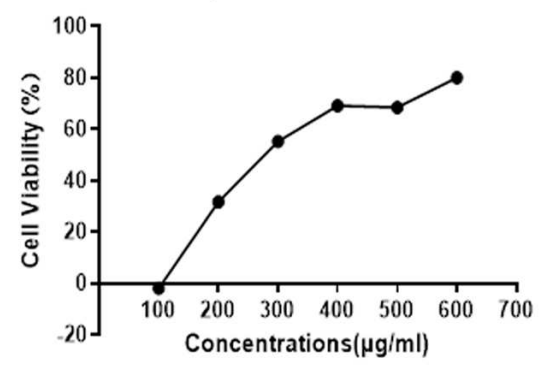

\section{Figure 1}

Cytotoxicity of CR and cell viability rate were evaluated by cell viability assay. (a )The CC50 of CR were determined by cell viability assay in Vero-E6 cells. (b ) The CC50 of CR were determined by cell viability assay in Hela cells. (c) CPE reduction assay examining the effects of CR on ZIKV-infected Vero-E6 cells 
compared with the control and model. Different modes of administration, the cell survival rates of CR on ZIKV were determined by cell viability assay at $48 \mathrm{~h}$ post-infection. (d) Premixed administration: Drugs

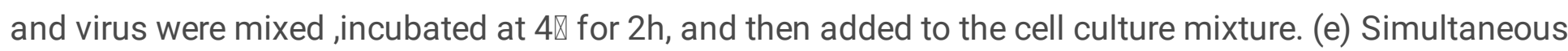
administrations: Drugs and virus were mixed and immediately added to the cell culture. $(f)$ Therapeutic administration:Drugs were added at $2 \mathrm{hpi}$ by the virus to MDCK cells. $\mathrm{n}=3$,each concentration conducted in tripicate.

a

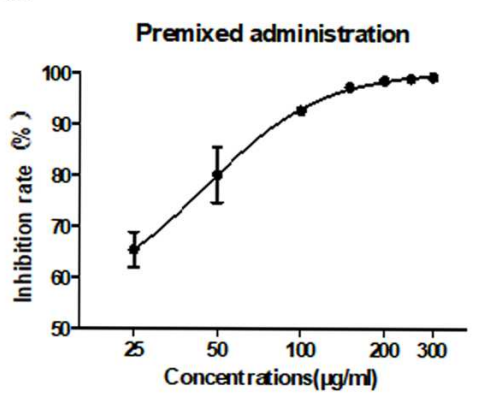

d

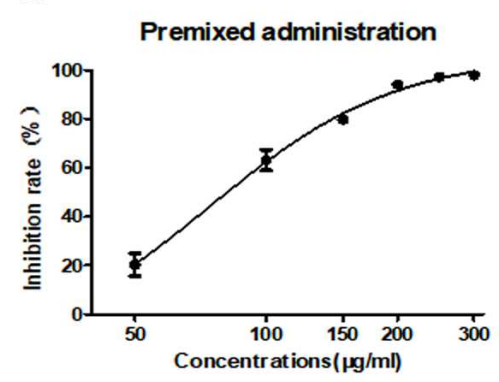

g

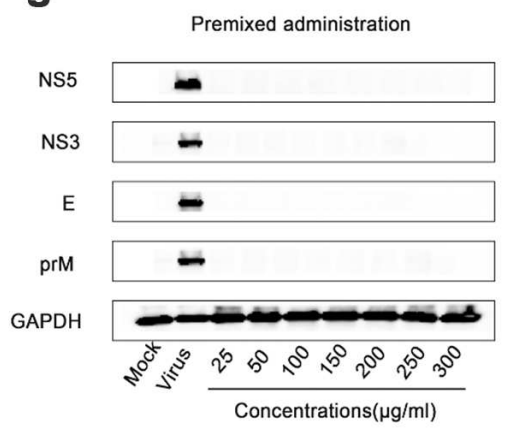

b

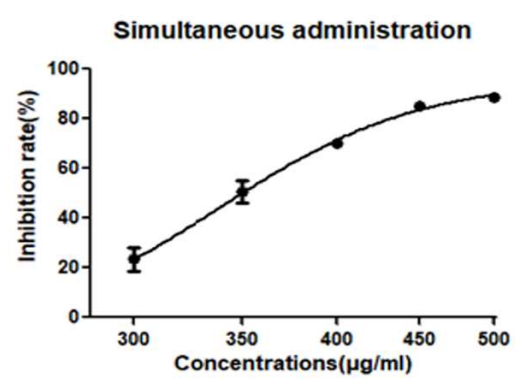

e

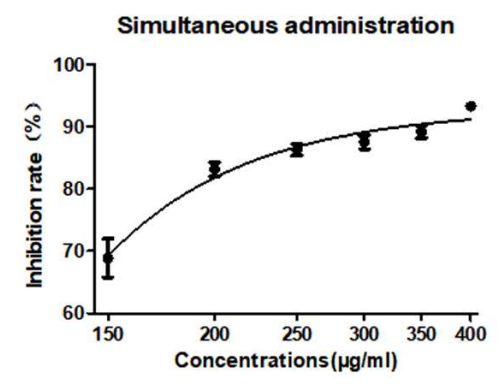

h

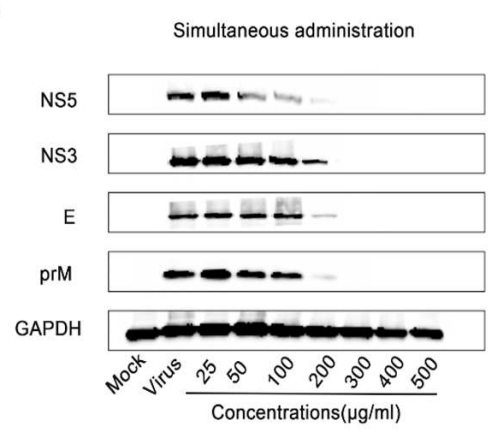

C

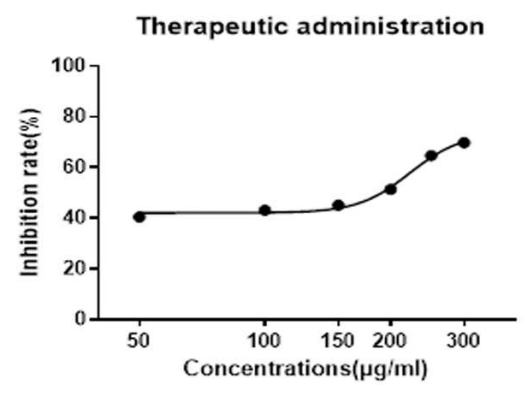

f

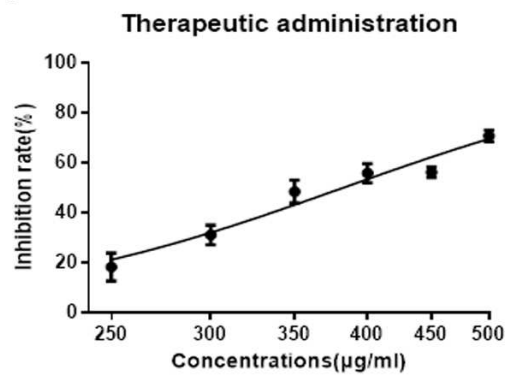

i

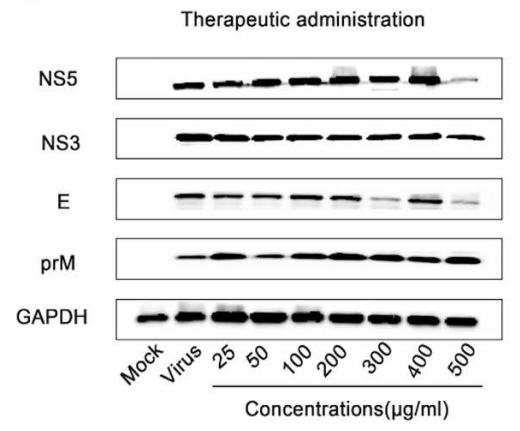


Antiviral activities of CR by qRT-PCR analysis and Western blot assays. (a-c) Inhibitory effects of various concentrations of $\mathrm{CR}$ administered in three infection protocols on ZIKV-infected Vero-E6 cells by qRT-PCR assay, $n=3$,each concentration conducted in tripicate. (d-f) Inhibitory effects of various concentrations of CR administered in three infection protocols on ZIKV-infected Hela cells by qRT-PCR assay. $n=3$,each concentration conducted in tripicate. ( $\mathrm{g}-\mathrm{j})$ Level ZIKV proteins expression effects of various concentrations of CR administered in three infection protocols on ZIKV-infected Hela cells by Western-blot
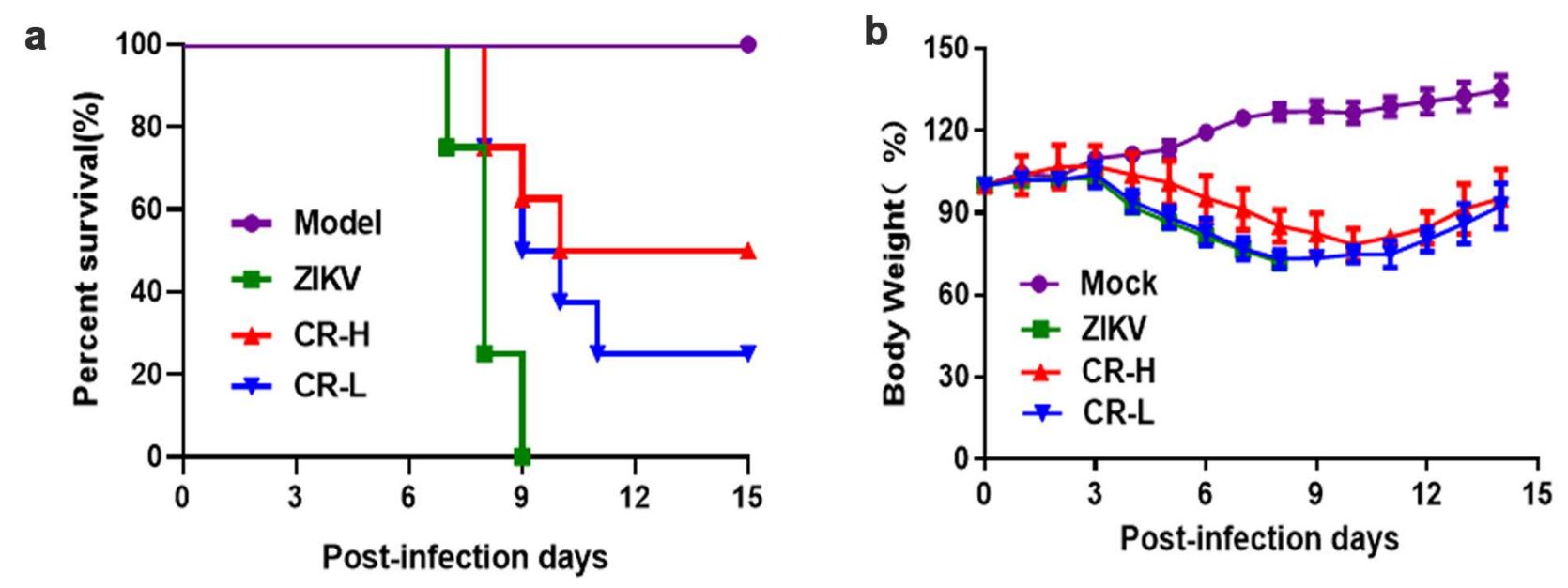

C

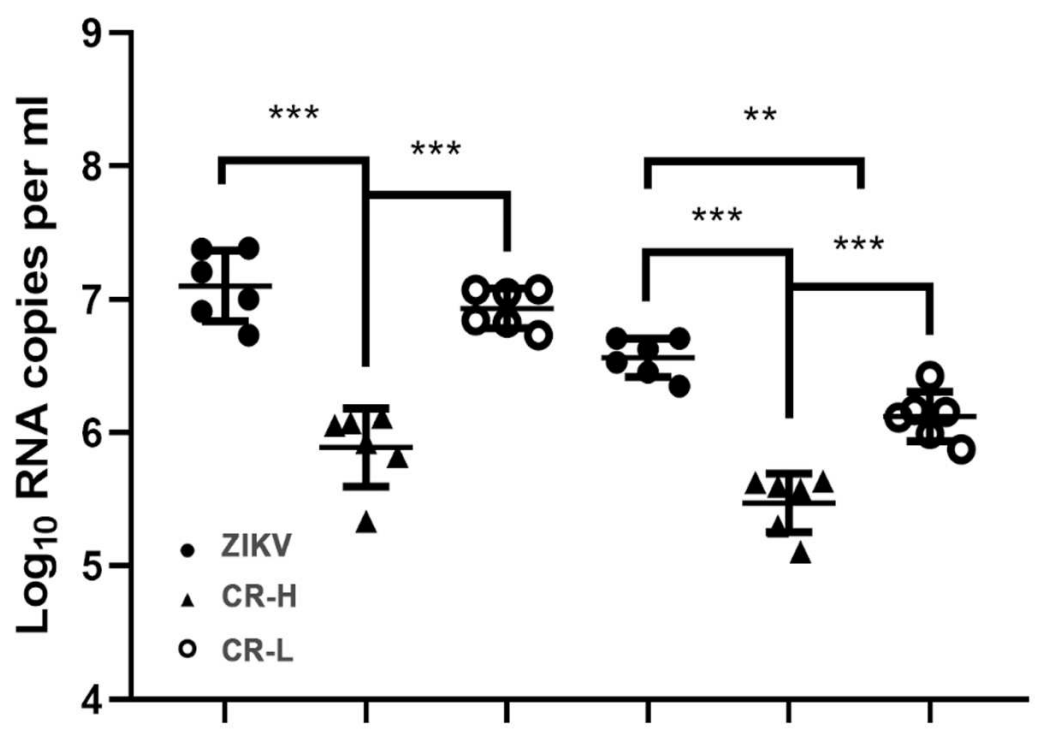

Day 3

Day 5 


\section{Figure 3}

Therapeutic effect of CR on ZIKV in AG6 mice. (a) Mice were infected with 3.3×106 pfu of ZIKV,the survival rate and survival days were monitored until 15 days by receiveing oral administration of the saline or CR $(900 \mathrm{mg} / \mathrm{ml}, 450 \mathrm{mg} / \mathrm{ml})$. (b) Mice were infected with $3.3 \times 106$ pfu of ZIKV, the body weight lossing were monitored until 15 days by receiveing oral administration of the saline or CR ( 900 $\mathrm{mg} / \mathrm{ml}, 450 \mathrm{mg} / \mathrm{ml}$ ). (c) Blood samples were collected on day 3 and day 5 p.i and subjected to qRT-PCR and detected viral RNA replication level. $n=6$, date are represented as the mean $\pm S . D . * P \llbracket 0.05 \llbracket \star * P \llbracket 0.01 \rrbracket$ $\star * * P \otimes 0.001$. 
a

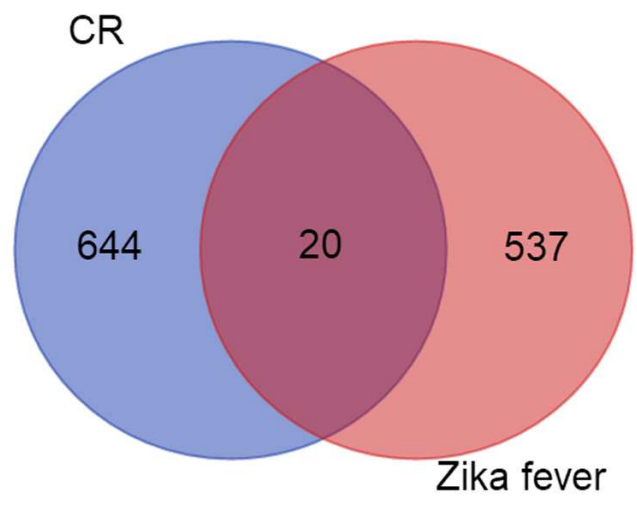

b

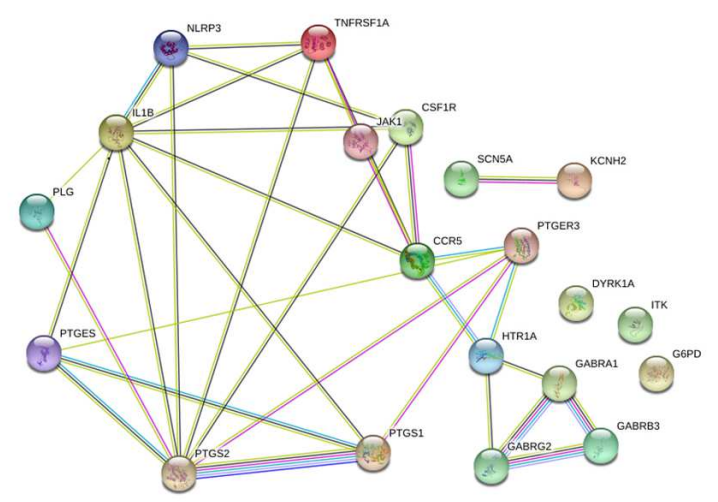

C

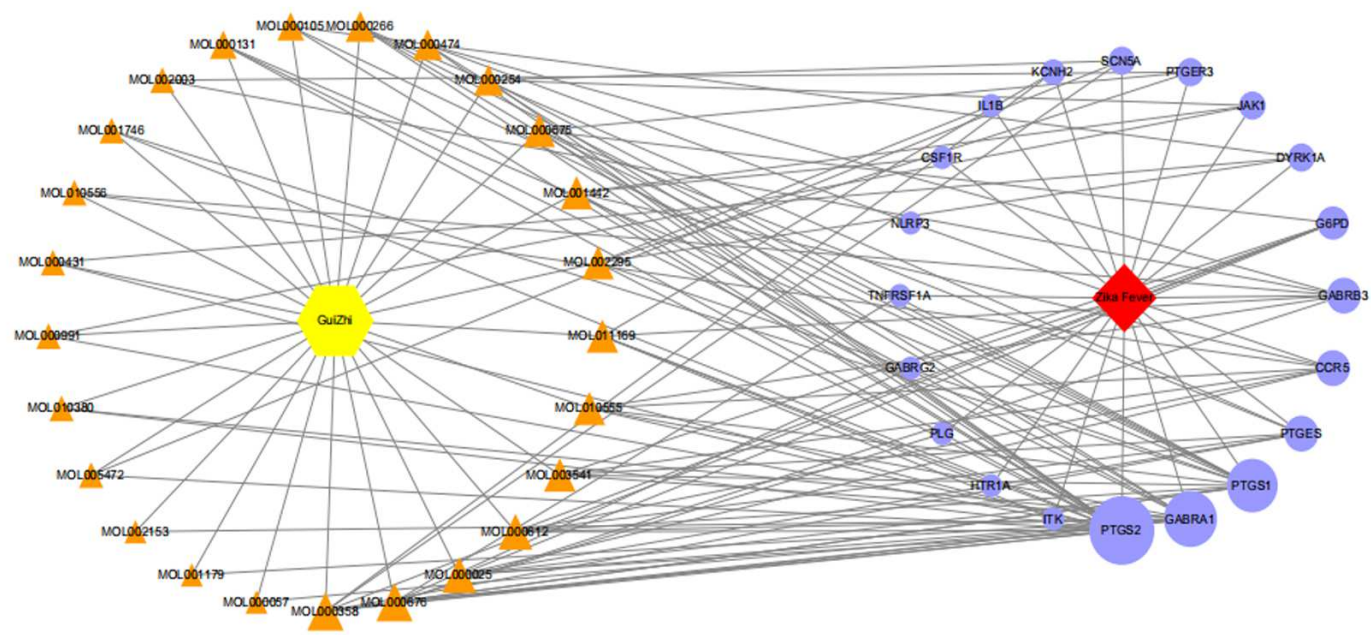

\section{Figure 4}

Screening of active compounds and potential targets. (a) The 20 matching targets of the related targets in CR on Zikv fever. (b) The PPI network was constructed by 20 nodes and 30 edges in the String database. (c) The Drug-Target-Disease network that consists of 48 nodes and 136 edges. yellow nodes denote the $\mathrm{CR}$, red nodes denote the disease-Zika fever,orange nodes denote the active ingredients and 
blue nodes denote the potential targets,respectively. Node size is relative to the degree, and the edges demonstrate the interaction between nodes.
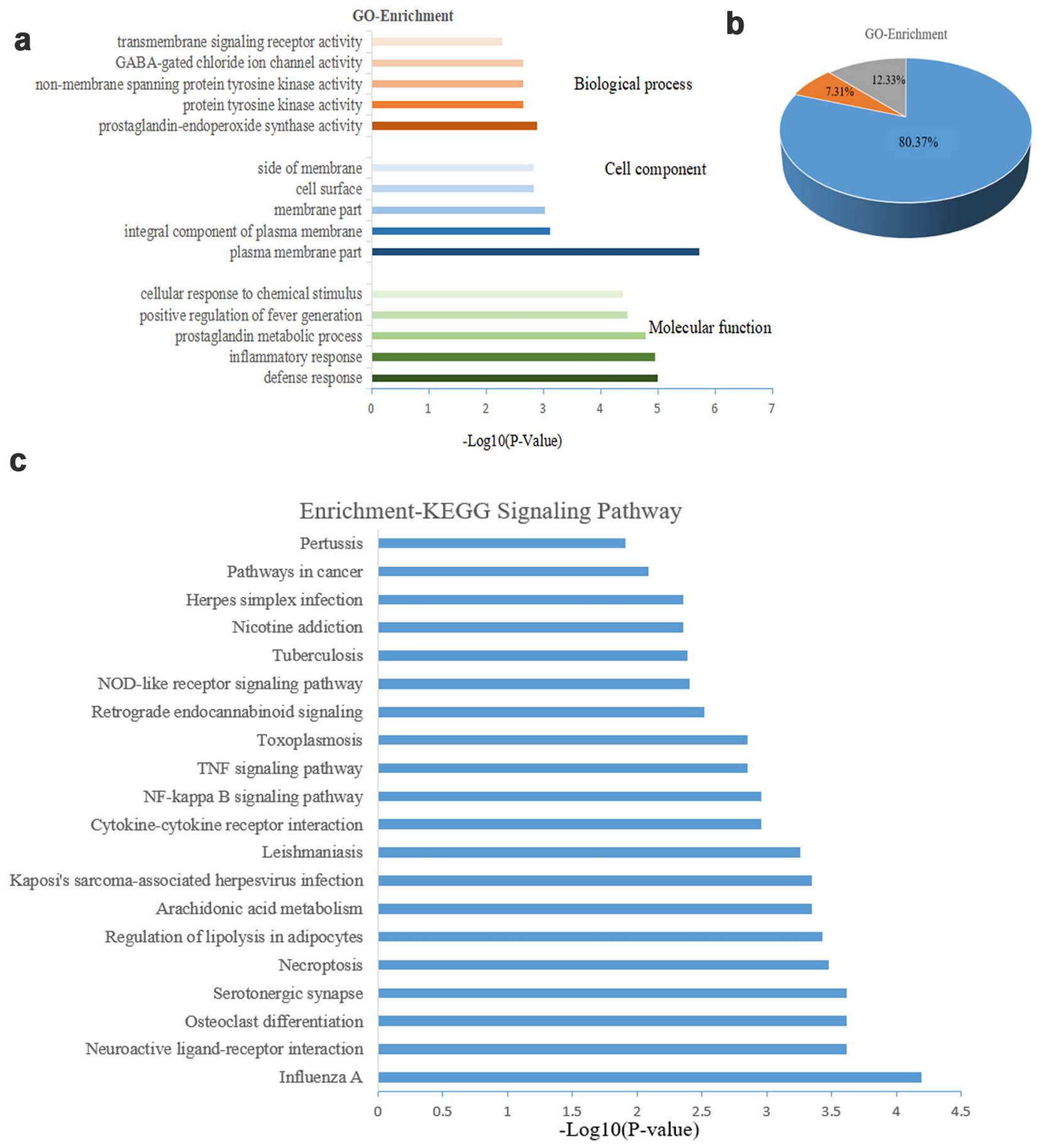

Figure 5

GO enrichment and KEGG enrichment analysis of overlapping targets. (a) Top5 significantly enriched terms are shown in the biological process, cell component, and molecular function from STRING 
database. (b) Percentage of three items are shown by GO enrichment analysis. (c) The top 20 remarkably enriched pathways are shown.

\section{Supplementary Files}

This is a list of supplementary files associated with this preprint. Click to download.

- Table1.pdf

- Table2.pdf

- Table3.pdf 